\title{
Clustering Design of Biological Community Diversity in City Landscape Based on MapGIS
}

\author{
Xin Zheng ${ }^{1}$, Wei $\mathrm{Li}^{2}$ \\ ${ }^{1}$ School of Art Design, Jilin Jianzhu University, Changchun,130118, China \\ ${ }^{2}$ School of Architecture and City Plan, Jilin Jianzhu University, Changchun, 130118, China \\ xin_zheng2014@yeah.net
}

Keywords: Landscape design, Biotic community, Lagrange, Secondary development, MapGIS software, Operator.

\begin{abstract}
In order to realize the sustainable development of cold city waterfront Landscape and construct the low-carbon city, this paper proposes a new biological community diversity method in the scenic area planning and design. In order to verify the validity of the method, this paper established the clustering mathematical model of biological community diversity, and uses the Lagrange operator to optimize the best path. In the cold city waterfront landscape planning and design process, this paper uses the MapGIS to complete the diversity layout of landscape biological communities, and uses the programming methods to do secondary development on MapGIS, which realizes the diversity cluster of biotic community and provides a new computer method for the study on cold city waterfront landscape design.
\end{abstract}

\section{Introduction}

Micro green is a small scale public open space for the public, and it can provide a relaxing and gathering for city residents near the site. Micro green includes small park green space, street green space or attached green space and all kinds of buildings etc. [1, 2]. One of the important ways of sustainable development for cold city waterfront Landscape is the protection of green space, increasing the green area and improving the green level to ease and improve the city's ecological environment. This paper introduces a new kind of design method in the design process of cold city landscape. The overall design framework is as shown in Figure 1.

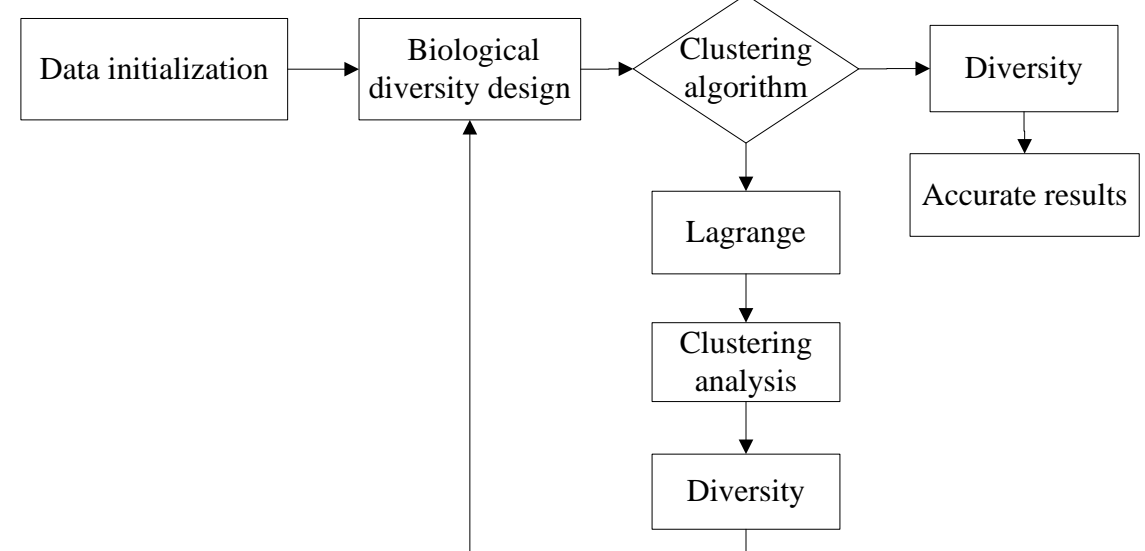

Fig.1: The waterfront landscape design framework

Figure 1 shows the overall framework of cold city waterfront landscape design. In the design process of landscape this paper introduces biotic community's diversity, and uses clustering algorithm to identify the diversity. It uses the Lagrange operator to optimize the design of diversity, and uses feedback regulation way to analyze the design effect. If biological diversity meets the low carbon environmental protection design, it will directly output accurate results $[3,4]$; if not meet the design regulation, it will re-cluster analysis to complete the design of biological community structure. 


\section{The Cluster Mathematical Model and Algorithm Design of Biotic Community Diversity}

In order to test all kinds of curtain wall ventilation speed, this paper designs a new orifice meter method. This method goes through the orifice plate to increasing the flow velocity, after the airflow velocity is increased, the pressure difference before and after orifice plate will also be increased, so we can go through measuring the differential pressure to measure the size of air flow, so as to test the ventilation speed.

The clustering algorithm of biological diversity is through clustering of $n$ variable to find the best optimization path, and then the membership space is divided, and the sum is 1 , the $n$ variable $q_{1}, q_{2}, q_{3}, \cdots, q_{i}$ are divided into $q$ math combination, finally calculate the cluster centers for each array [5-7]. Its expression is as follows:

$$
\sum_{i=1}^{q} v_{i q}=1, \forall q=1, \ldots, n \text {. }
$$

Among them, $v$ is the cluster center. The general function of cluster algorithm analysis can be expressed as:

$$
M\left(V, z_{1}, \cdots, z_{c}\right)=\sum_{i=1}^{z} M_{i}=\sum_{i=1}^{z} \sum_{q}^{n} v_{i q}^{k} d_{i q}^{2} .
$$

Among them, $v_{i q}$ is between 0 and $1, z$ is the clustering center, $d_{i q}^{2}$ is the Euclidean distance square between distance center and $q$ data point, $k$ is the weighted index which is greater or equal to 1.

$$
\begin{array}{r}
\bar{M}\left(V, z_{1}, \cdots, z_{c}, \alpha_{1}, \cdots, \alpha_{n}\right)=M\left(V, z_{1}, \cdots, z_{c}\right)+\sum_{q=1}^{n} \alpha_{q}\left(\sum_{i=1}^{z} v_{i q}-1\right) \\
=\sum_{i=1}^{z} \sum_{q}^{n} v_{i q}^{k} d_{i q}^{2}+\sum_{q=1}^{n} \alpha_{q}\left(\sum_{i=1}^{z} v_{i q}-1\right) .
\end{array}
$$

Among them, $\alpha_{1}, \cdots, \alpha_{n}$ is Lagrange operator, and the expression of constraint conditions are as follows:

$$
\mathrm{Z}_{i}=\frac{\sum_{q=1}^{n} v^{k}{ }_{i q} \alpha_{q}}{\sum_{q=1}^{n} v_{i q}^{k}}
$$

In order to achieve the above algorithm, this paper uses programming method to do secondary development on MapGIS, and the main program is as follows:

int clusterOfTuple(Tuple means[],Tuple tuple)

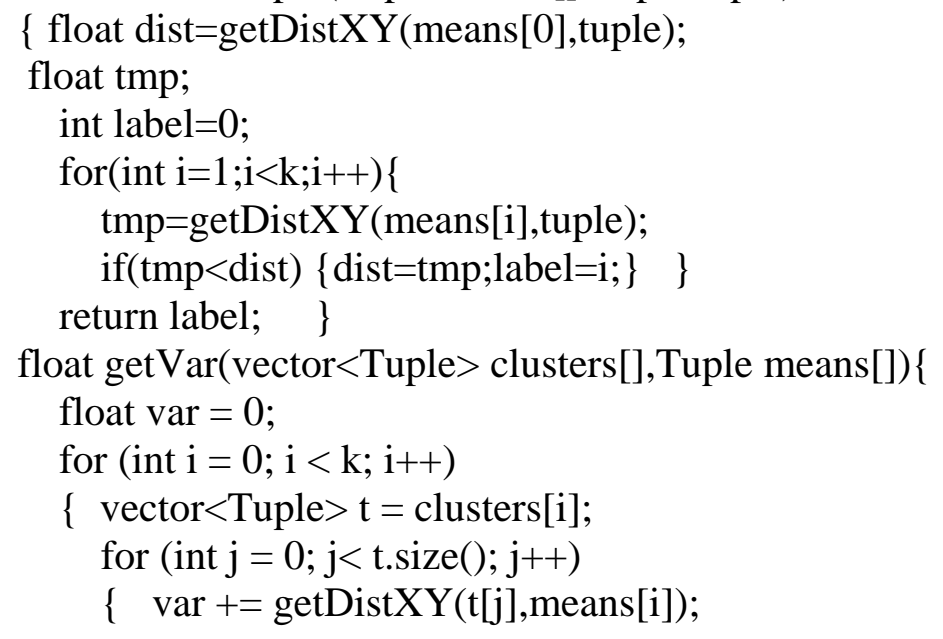




\author{
\} $\}$ \\ return var; \}
}

\title{
Study on The Cold City Waterfront Landscape Micro Green Area Design based on MapGIS
}

In order to verify the effectiveness and reliability of biological community diversity clustering algorithm designed in second section, this paper uses MapGIS software to design the waterfront landscape micro green area in the cold city, so as to achieve the low carbon and environmental protection effect $[8,9]$. The preliminary planning diagram is as follows:

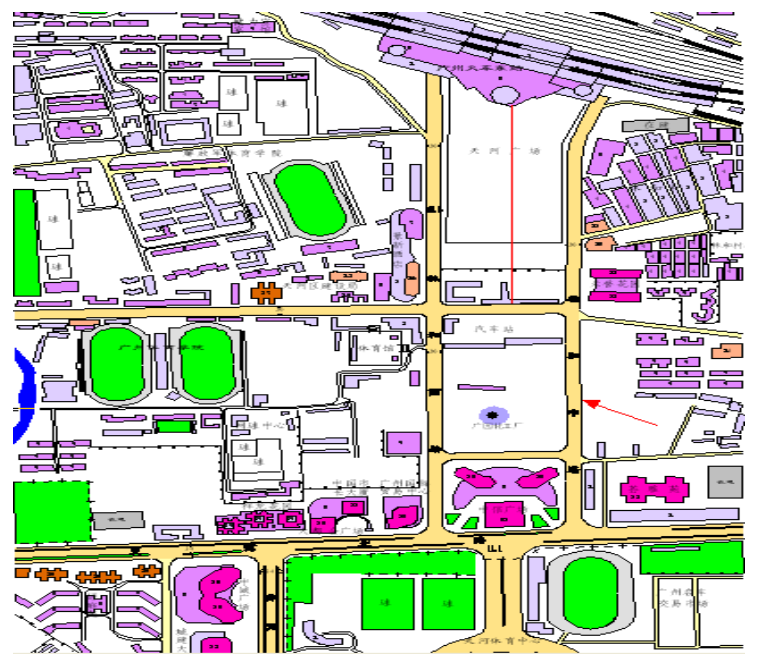

Fig.2: MapGIS preliminary planning map

Figure 2 shows the preliminary planning map using MapGIS software to do space planning on landscape. Spatial analysis is one of the important functions of the GIS system, space analysis object is generally a large amount of data which is related to spatial, and the data includes spatial location and attribute [10]. Spatial coordinates is to describe the position and geometry of space, and attribute is used to describe the diversity and low carbon and environmental protection situation of biological community. As shown in Figure 3 it represents pollution range of chemical factory.

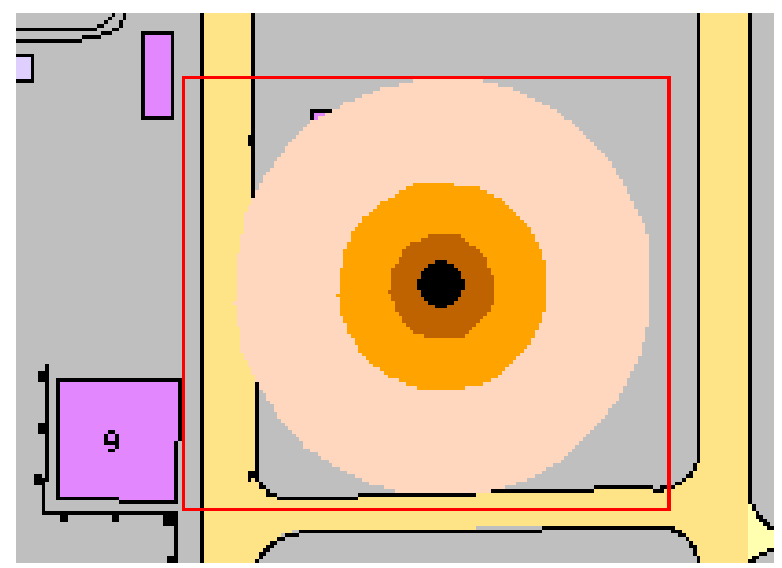

Fig.3: The pollution range of chemical plant

Figure 3 shows using MapGIS to marker the pollution range of chemical plant. In the waterfront landscape design, pollution is one of the main factors to consider. So the pollution planning needs to be controlled in the scope [11]. And it uses layers to describe the scope. 


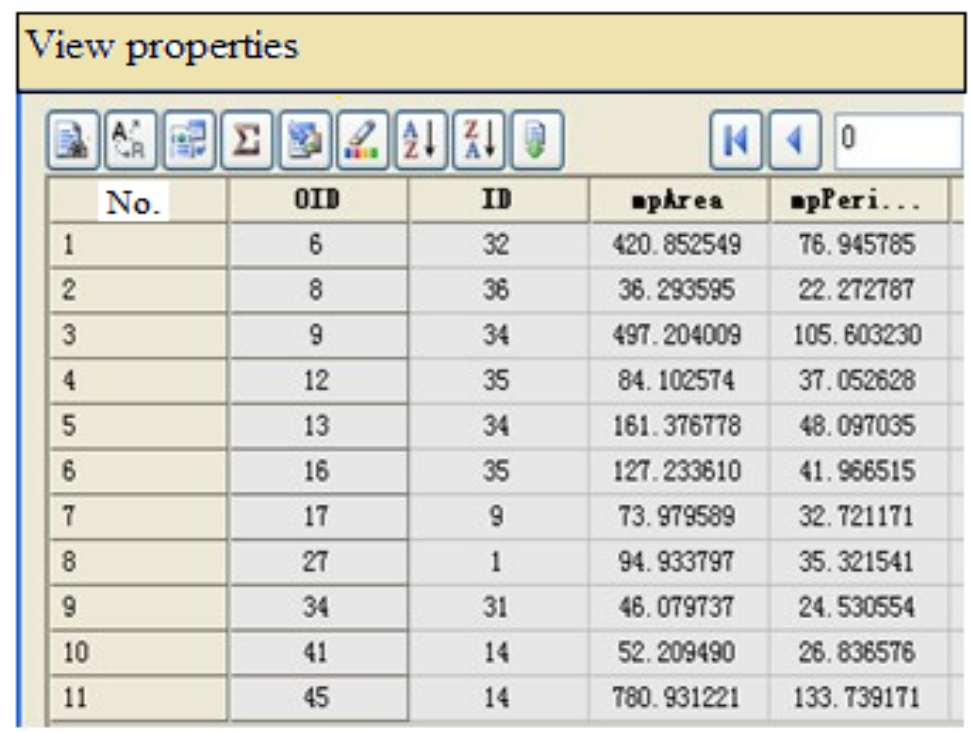

Fig.4: Biodiversity layer properties

In order to design the diversity of biological community, it needs to establish the layers of pollution zone, micro green area and square [12]. The layer mathematical data is as shown in Figure 4. This study designed 300 layers, and the planning path is as shown in Figure 5.

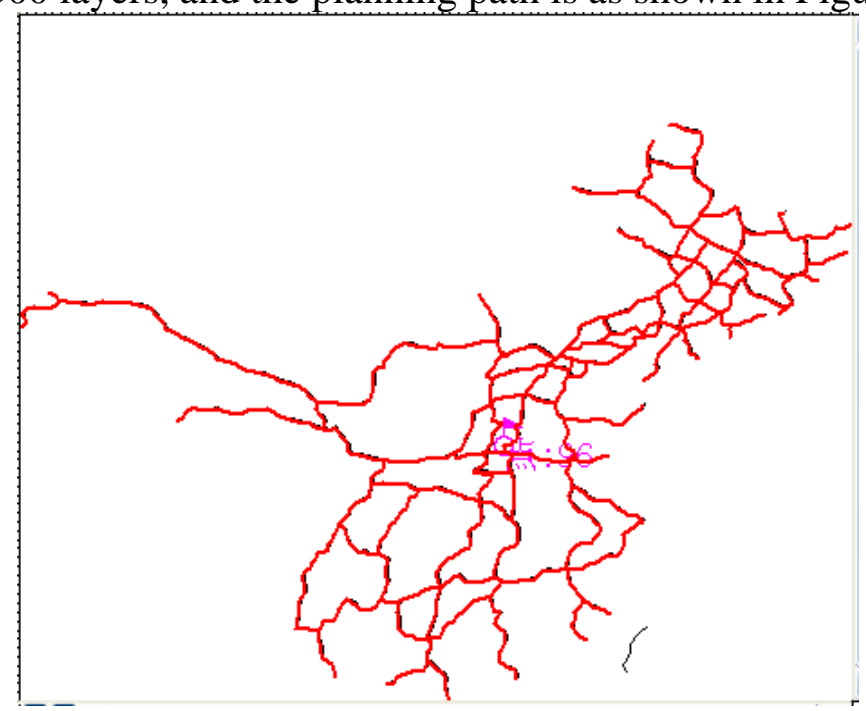

Fig.5: Biological diversity path planning

Figure 5 shows the diversity planning path of biological community [13]. From the chart it can be seen, in a number of landscape design path, it plans the main design line, at the intersection of route it can design the micro green area, and the detailed design is as shown in Figure 6.

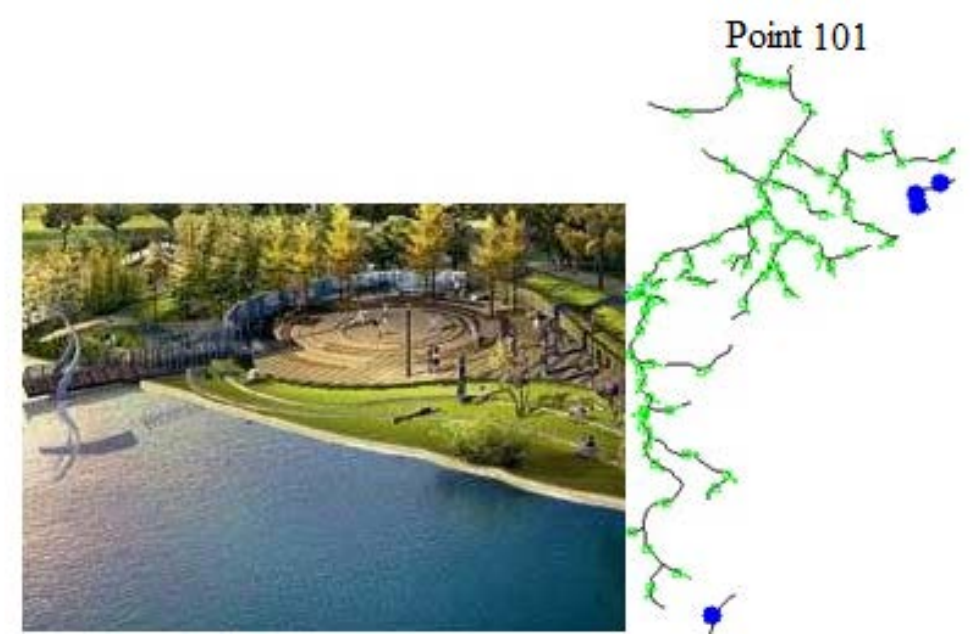

Fig.6: Biodiversity waterfront micro green area design 
Figure 6 shows the detailed case design of waterfront city micro green area in the intersection point of two paths [14]. This method can design all the micro green area, finally it uses clustering analysis algorithm to calculate the attribute as shown in Figure 7.

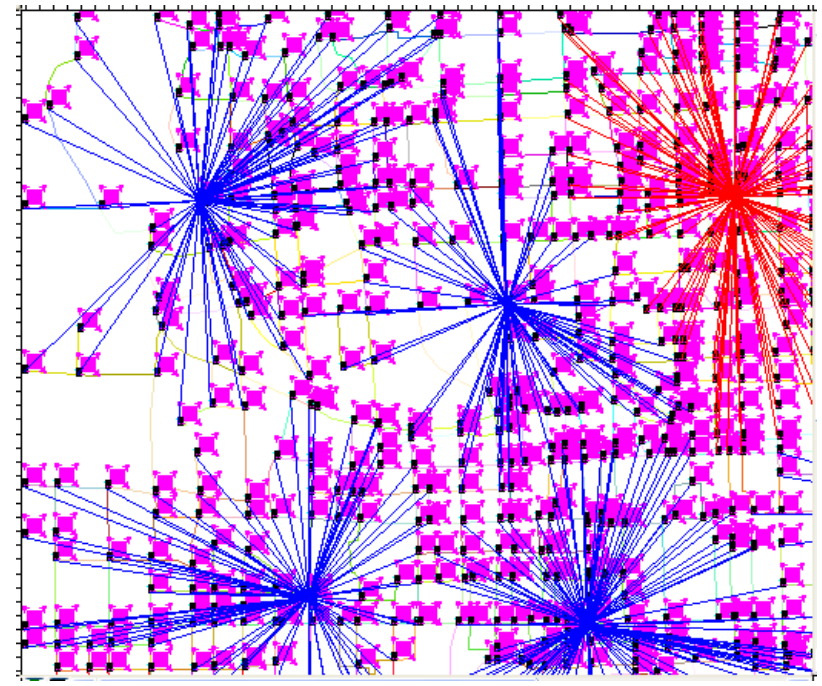

Fig.7: Biodiversity micro green area cluster design

As shown in Figure 7, it represents biodiversity micro green area cluster design. The basic principle is according to the property of the sample itself, by the method of mathematics, based on some similarity or difference index, it can quantitative determine the relationship between samples, and according to this kind of relation to cluster the sample [15]. Through the cluster computing, it can get the ecological relationship between micro green area, in the landscape design it can merge two pieces of similar attribute micro green area, which is convenient for landscape planning and design.

\section{Summary}

On the basis of cluster mathematical model of biological community, this paper puts forward a new method of low carbon environment-friendly city waterfront landscape design. This method uses the Lagrange operator to optimize the clustering of biological communities, and uses the programming way to realize cluster path, which improves the efficiency of landscape design. This paper uses MapGIS to do waterfront landscape design of the cold city micro green area, and does secondary development on the clustering calculation of biological community diversity, finally the similar attribute biological community point is obtained, which provides a theoretical reference for the design of cold city waterfront landscape.

\section{References}

[1] Y.Y. Liang, X.K. Chen, H.Y. Li. The cases and experience of South Korean city river ecological restoration. Water resources protection, 2013, 4(2): 20-23.

[2] Y. Huang, L.Y. Long. Exploration of regional waterfront landscape design strategy. Anhui Agricultural Science Bulletin, 2013, 4(1): 12-15.

[3] P.Y. Li, X.D. Li, Li Yi. The flow history and culture -the town planning of Lijiang, Hongcun and Suzhou. Jiangsu architecture, 2013, 4(2): 56-59.

[4] J. Wang. Discussion on the waterfront landscape design. Youth, 2013, 4(2): 78-80.

[5] D.W. Lu, J.G. Wen. Landscape images of the waterfront space -taking Suzhou Jinji Lake landscape design as an example. Construction technology, 2013, 2(2): 53-56.

[6] J.Q. Zou, D.Q. Song. Application of auantitative AHP model in the city residential plant landscape evaluation. Journal of Jinling Institute of Technology, 2012, 4(2): 91-93.

[7] J. Lv Juan. Preliminary study on city waterfront landscape design. Chinese conservancy, 2013, 4(1): 23-26. 
[8] D.F. Lu. University campus plant landscape evaluation model and its application. Journal of Fujian Forestry College, 2012, 4(31): 67-69.

[9] X. Ding. City waterfront plant landscape. Modern agricultural science and technology, 2012, 4(20): 46-48.

[10] G.J. Pang, J. Wang. On the water landscape design. Shanxi architecture, 2012, 4(5): 112-115.

[11] K.J. Yu. The city landscape as a living system - the 2010 Shanghai Expo Houtan Park. Journal of architecture, 2013, 4(5): 104-106.

[12] H.Y. Liu, C. Su, H. Luan. Yangzhou water culture industry planning and its form of industry innovation. Theory of Jiangsu, 2013, 5(2): 45-48.

[13] K. Chen, Y.H. Wu, H. Li, R.J. Bi. Plants in waterfront landscape design. Guangxi tropical agriculture, 2013, 2(8): 52-55.

[14] H.S. Ruan. Study on the types of modern city waterfront landscape planning. Anhui Agricultural Science Bulletin (half monthly), 2013, 4(2): 112-115.

[15] Q.J. Jiang, Z.W. Chen, A.X. Li. Analysis of the city waterfront landscape design. Henan building materials, 2012, 3(3): 78-80. 